\title{
Prognostic Value of Lymphocyte-to-Monocyte Ratio in Canine High-Grade Lymphoma Cases
}

\author{
Michihito Tagawa $^{1 *}$, Genya Shimbo ${ }^{1,2}$, Kotaro Matsumoto ${ }^{3}$ and Kazuro Miyahara ${ }^{1}$ \\ ${ }^{1}$ Veterinary Medical Center, Obihiro University of Agriculture and Veterinary Medicine, Inada, Obihiro, Hokkaido 080-8555, Japan \\ ${ }^{2}$ Veterinary Teaching Hospital, Faculty of Veterinary Medicine, Hokkaido University, Kita-ku, N18 W9, Sapporo, Hokkaido, 060-0818, Japan \\ ${ }^{3}$ Department of Veterinary Medicine, Division of Clinical Veterinary Medicine, Obihiro University of Agriculture and Veterinary Medicine, Inada, \\ Obihiro, Hokkaido 080-8555, Japan \\ *Corresponding author's E-mail: mtagawa@obihiro.ac.jp; ORCID: 0000-0003-1645-4413
}

\begin{abstract}
The Lymphocyte-to-Monocyte Ratio (LMR) has been described as a useful prognostic marker for human patients with various cancers and dogs with diffuse large B-cell lymphoma. The objective of this study was to determine whether the LMR could predict disease outcome as measured by the Time To Progression (TTP) and Overall Survival (OS) of dogs with different types of high-grade lymphoma. The medical records of 43 dogs diagnosed with high-grade lymphoma at the Veterinary Medical Center of Obihiro University of Agriculture and Veterinary Medicine between 2013 and 2018, were retrospectively analyzed. Receiver Operating Characteristic (ROC) curve analysis was used to determine the optimal LMR cutoff values. The prognostic influence of the LMR and other clinicopathological data on TTP and OS was studied by Kaplan-Meier curves. To identify the independent prognostic factors, univariate and multivariate Cox proportional analyses were used. The optimal cutoff value of the LMR was 0.7, which corresponded to the maximum sensitivity (0.727) and specificity (0.762) of the LMR for predicting the median days of OS with ROC analysis (area under the curve, 0.794). Log-rank tests showed that dogs with a high LMR had significantly longer TTP and OS than dogs with a low LMR. Moreover, immunophenotype, body weight, treatment regimen and response to treatment were significantly associated with TTP and OS. In multivariate analysis, treatment and response to treatment were independent risk factors for TTP. Moreover, the LMR, treatment regimen and response to treatment were independent predictors of OS.
\end{abstract}

Key words: Dog, Lymphocyte to monocyte ratio, Lymphoma, Prognosis

\section{INTRODUCTION}

Canine lymphoma is a prevalent malignancy in dogs, representing almost $80 \%$ of all hematopoietic cancers (Argyle and Pecceu, 2016). Combination chemotherapy regimen consisting of Cyclophosphamide, Doxorubicin, Vincristine, and Prednisone (known as CHOP), is often recommended as the standard therapy for dogs with lymphoma. Initially, the disease is highly responsive to CHOP-based protocols with first remission rates of $85 \%$ or greater. However, drug resistance occurs in most cases, resulted in disease recurrence and overall median survival times are limited to 10-12 months (Vail et al., 2013). Several factors assessed at diagnosis have been proposed as predictors of clinical outcome in dogs with high-grade lymphoma, including anatomical form, immunophenotype, clinical stage and substage (Teske et al., 1994; Vail et al., 2013). The gastrointestinal form of lymphoma has a poorer prognosis compared to the multicentric form (Vail et al., 2013). The two prognostic factors, T-cell immunophenotype and substage b are most consistently identified as poor prognostic factors (Childress et al., 2018).

Lymphocytes mediate humoral and cellular antitumor immune responses, whereas monocytes promote cancer progression through local immune suppression and angiogenesis (Parihar et al., 2010; Whiteside, 2005). In human medicine, low lymphocyte and high monocyte counts have been observed in patients with advanced cancer which are associated with poor prognosis in patients with various malignancies (Ray-Coquard et al., 2009; Aoki et al., 2013). Recently, the prognostic value of the Lymphocyte-to-Monocyte Ratio (LMR), which is calculated by dividing the absolute lymphocyte count by the absolute monocyte count, has been reported for a number of different malignancies, including hematopoietic tumors (Failing et al., 2017). Moreover, it has been reported that a low LMR may indicate an unfavorable prognosis in dogs with diffuse large B-cell lymphoma (Marconato et al., 2015; Davies et al., 2018).

However, there are many different canine lymphoma types, such as multicentric, gastrointestinal, skin forms and Bor T-cell immunophenotypes. It is unclear whether the LMR behaves as a prognostic factor for these different types of lymphomas or not. The present study retrospectively analyzed the medical records of dogs with high-grade lymphoma to investigate the prognostic value of the LMR at diagnosis with respect to the Time To Progression (TTP) and Overall Survival (OS). 


\section{MATERIALS AND METHODS}

\section{Ethical approval}

This study was subjected to ethical examination at the Veterinary Medical Center, Obihiro University of Agriculture and Veterinary Medicine (VMC-OUAVM) and was approved.

\section{Study population and diagnosis}

This retrospective study reviewed Medical records of dogs with high-grade lymphomas, classified according to the updated Kiel classification (Fournel-Fleury et al., 1997), which were cytologically or histologically confirmed at the VMC-OUAVM, Japan between 2013 and 2018. The dogs that had not received lymphoma specific treatment regimens, including prednisolone or any other cytotoxic agents, were excluded from the study. Dogs were clinically staged at the time of diagnosis according to the WHO staging system (Owen, 1980), based on findings of three-view thoracic radiography, abdominal ultrasound, and hematological examinations. The immunophenotype of tumor samples, obtained by biopsy or fine-needle aspiration, was determined by immunohistochemistry, flow cytometry, or Polymerase chain reaction for Antigen Receptor Rearrangement (PARR). Briefly, the lymphoma immunophenotyping evaluated histomorphological features as well as CD3 and CD20 expression by tumor cells through immunohistochemical analysis. These procedures had been conducted at the OUAVM Pathological Laboratory or a commercial laboratory. Flow cytometric analysis, which was previously described by Tagawa et al. (2018), was performed by analyzing morphological scattergrams and CD4/CD8 (clone YKIX302.9/YCATE55.9; AbD Serotec, Raleigh, NC, USA) and CD21 (clone CA2.1D6; AbD Serotec, Kidlington, United Kingdom) expression using BD FACS Cant and FACS Diva software (BD Biosciences, San Jose, CA, USA). PARR was performed as previously described (Lana et al., 2006).

\section{Data sampling}

All the clinicopathological data including age, breed, sex, body weight, complete blood count (CBC) and serum biochemistry profiles, for each case, were retrieved from the medical records of VMC-OUAVM. The CBC was measured using automated hematology analyzer (Celltac $\alpha$, MEK-6458; Nihon Kohden, Tokyo, Japan), and serum biochemistry analysis was performed using VETTEST analyzer (IDEXX Laboratories, Tokyo, Japan). Blood smears were reviewed by trained medical technologists and, in some cases, a veterinarian. Leukocyte differential counts were performed manually by counting 100 cells. The LMR was calculated as the ratio of the absolute count of lymphocytes to monocytes in peripheral blood samples obtained on the day of diagnosis. Neoplastic cells that were larger than a neutrophil and had a nucleus that was more than 1.5 times the size of a red blood cell, with loose chromatin and evident nucleoli were excluded from the calculation (Graff et al., 2014).

\section{Response assessment}

The definitions for the response to treatment and relapse criteria used at each visit were described by Vail et al. (2010) based on physical examination, radiography, abdominal ultrasound, or hematologic examination at the clinician's discretion. Complete Remission (CR) was defined as the resolution of all measurable parameters of the disease. Partial Remission (PR) was defined as a $30 \%$ or more reduction in all measurable lesions. Dogs did not achieve CR or PR were categorized as No Remission (NR). TTP was defined as the elapsed time from the day of diagnosis to the day of stage progression. OS was defined as the time interval between the date of diagnosis and death, caused by any reason, or euthanasia. Dogs that remained alive at the end of the follow-up period or that were lost to follow-up were censored from the OS analysis. In case of relapse, rescue treatment was offered to the owners.

\section{Statistical analysis}

Receiver Operating Characteristic (ROC) curve analysis was used to determine the optimal LMR cut-off values for the prediction of a survival time exceeding median days. A minimum Area Under the Curve (AUC) of 0.7 was required to consider for the ROC model. The variables assessed for prognostic significance for the TTP and OS were as follows: age ( $\geq$ or $<10$ years), sex (male or female), weight ( $\geq$ or $<10 \mathrm{~kg}$ ), WHO stage (I-V), substage (a or b), anatomical form (multicentric, alimentary, cutaneous, and other form), immunophenotype (B-cell, T-cell, or null type), hypercalcemia (presence or absence at presentation), anemia (presence or absence at presentation), lymphopenia ( $\geq$ or $<1000$ cells $/ \mu l$ ), monocytosis $(\geq$ or $<1400$ cells/ $\mu \mathrm{l}$ ), treatment regimen (CHOP-based, single agent, or prednisolone alone), response to treatment (CR, PR, or NR), prior prednisolone administration (yes or no) and the LMR (high or low). A Kaplan-Meier analysis with log-rank test was performed to estimate the TTP and OS. Univariate and multivariate Cox proportional analyses were used to identify independent prognostic factors. Variables with $\mathrm{p} \leq 0.1$ in the univariate analysis were then entered into the multivariate analysis. All analyses were performed using JMP 13 (SAS Institute Inc., Cary, NC, USA). Differences were considered statistically significant at a p-value $<0.05$. 


\section{Patient characteristics and cut-off point determination}

A total of 62 cases were identified for initial review and 19 dogs were excluded from the study because of the lack of clinical data or not meeting the eligibility criteria. Finally, 43 dogs with high-grade lymphomas were included in the present study. The Breeds included miniature Dachshund (7 dogs), mixed breed (7 dogs), Golden Retriever (4 dogs), Miniature Schnauzer (3 dogs), Shiba (3 dogs), Labrador Retriever (2 dogs), Border Collie (2 dogs), Toy Poodle (2 dogs), and Shih Tzu (2 dogs). In addition, American Cocker Spaniel, English Cocker Spaniel, Flat-coated retriever, miniature Pinscher, Maltese, French Bulldog, Beagle, Papillon, Chihuahua, Shetland Sheepdog, and Pembroke Welsh Corgi included one dog each. Patient characteristics are summarized in table 1.

Table 1. Clinical characteristics and patient variables of 43 dogs diagnosed with high-grade lymphoma at Veterinary Medical Center of Obihiro University of Agriculture and Veterinary Medicine between 2013 and 2018.

\begin{tabular}{|c|c|c|}
\hline Characteristics & Case & Percentage (\%) \\
\hline Age, years; median (range) & $9(1-15)$ & \\
\hline$\geq 10$ years & 21 & 48.8 \\
\hline$<10$ years & 22 & 51.2 \\
\hline Body weight, kg; median (range) & $8.02(2.56-45.50)$ & \\
\hline$\geq 10 \mathrm{~kg}$ & 19 & 44.2 \\
\hline$<10 \mathrm{~kg}$ & 24 & 55.8 \\
\hline \multicolumn{3}{|l|}{ Sex } \\
\hline Male (Castrated) & $26(16)$ & $60.5(37.2)$ \\
\hline Female (Spayed) & $17(13)$ & $39.5(30.2)$ \\
\hline \multicolumn{3}{|l|}{ Stage } \\
\hline I & 3 & 7.0 \\
\hline II & 5 & 11.6 \\
\hline III & 10 & 23.3 \\
\hline IV & 16 & 37.2 \\
\hline $\mathrm{V}$ & 9 & 20.9 \\
\hline \multicolumn{3}{|l|}{ Substage } \\
\hline $\mathrm{a}$ & 17 & 39.5 \\
\hline $\mathrm{b}$ & 26 & 60.5 \\
\hline \multicolumn{3}{|l|}{ Immunophenotype* } \\
\hline B cell & 20 & 48.8 \\
\hline T cell & 17 & 41.5 \\
\hline Null type & 4 & 9.8 \\
\hline \multicolumn{3}{|l|}{ Anatomical form } \\
\hline Multicentric & 24 & 55.8 \\
\hline Alimentary & 11 & 25.6 \\
\hline Cutaneous & 5 & 11.6 \\
\hline Others (renal, CNS, mediastinal) & 3 & 7.0 \\
\hline \multicolumn{3}{|l|}{ Hypercalcemia } \\
\hline Yes & 4 & 9.3 \\
\hline No & 39 & 90.7 \\
\hline \multicolumn{3}{|l|}{ Presence of anemia } \\
\hline Yes & 16 & 37.2 \\
\hline No & 27 & 62.8 \\
\hline \multicolumn{3}{|l|}{ Lymphopenia } \\
\hline$\geq 1000 \mathrm{cells} / \mu \mathrm{l}$ & 25 & 58.1 \\
\hline$<1000$ cells $/ \mu 1$ & 18 & 41.9 \\
\hline \multicolumn{3}{|l|}{ Monocytosis } \\
\hline$\geq 1400$ cells $/ \mu 1$ & 26 & 60.5 \\
\hline$<1400$ cells $/ \mu 1$ & 17 & 39.5 \\
\hline \multicolumn{3}{|l|}{ Treatment } \\
\hline CHOP-based & 27 & 62.8 \\
\hline Single-agent & 10 & 23.3 \\
\hline Prednisolone & 6 & 14.0 \\
\hline \multicolumn{3}{|l|}{ Prior administration of prednisolone } \\
\hline Yes & 8 & 18.6 \\
\hline No & 35 & 81.4 \\
\hline
\end{tabular}


The median age was 9 years (ranged between 1-15 years) and median body weight was $8.02 \mathrm{~kg}$ (ranged between 2.56-45.50 kg). There were 26 male dogs (16 were castrated) and 17 female dogs (13 were spayed). According to the WHO staging criteria, three dogs were classified as stage I, five as stage II, 10 as stage III, 16 as stage IV and nine as stage V. Moreover, 17 and 26 dogs were classified as substage 'a' and 'b', respectively. Immunophenotyping was performed in $41 \mathrm{dogs}$, accomplished by immunohistochemistry of biopsy samples in 10 dogs and using flow cytometry and PARR of lymph node aspirate samples in 19 and $12 \mathrm{dogs}$, respectively. According to the obtained results from immunophenotyping of $41 \mathrm{dogs}, 20$ and 17 cases had B- and T-cell lymphoma, respectively. The remaining four cases were null type. Regarding the anatomical forms, 24 dogs were classified as multicentric, 11 dogs as alimentary (gastrointestinal and hepatosplenic) and five dogs as cutaneous. In addition, the renal, central nervous system, and mediastinal form were each found in one case. Hypercalcemia and anemia were observed in 4 and 16 dogs, respectively. Regarding treatment, 27 dogs were managed with a CHOP-based protocol, whereas six dogs had prednisolone monotherapy. The remaining 10 dogs were treated with other single agents (including L-asparaginase, doxorubicin, mitoxantrone, and lomustine). Only eight dogs were treated with prednisolone prior to sampling.

The median absolute lymphocyte concentration was 1176 cells/ $\mu 1$ (ranged between $0-12376$ cells/ $\mu 1$; reference range: 1000-4800 cells/ $\mu 1$; Mutz et al., 2015). Eighteen dogs had a lymphocyte count $<1000$ cells/ $\mu 1$. The median absolute monocyte concentration was 1547 cells/ $\mu$ l (ranged between $274-6200$ cells/ $\mu 1$; reference range: 100-1400 cells/ $\mu 1$; Mutz et al., 2015). Twenty-six dogs had a monocyte count $>1400$ cells/ $\mu 1$. The optimal LMR cut-off value was 0.7, which corresponded to the maximum sensitivity (0.727) and specificity (0.762) of the LMR for predicting the median days of OS with ROC analysis. The AUC of ROC for LMR was 0.794 ( $p=0.002$; Figure 1 ). An LMR $\geq 0.7$ (high LMR) was found in 22 dogs, and an LMR $<0.7$ (low LMR) was found in 21 dogs.

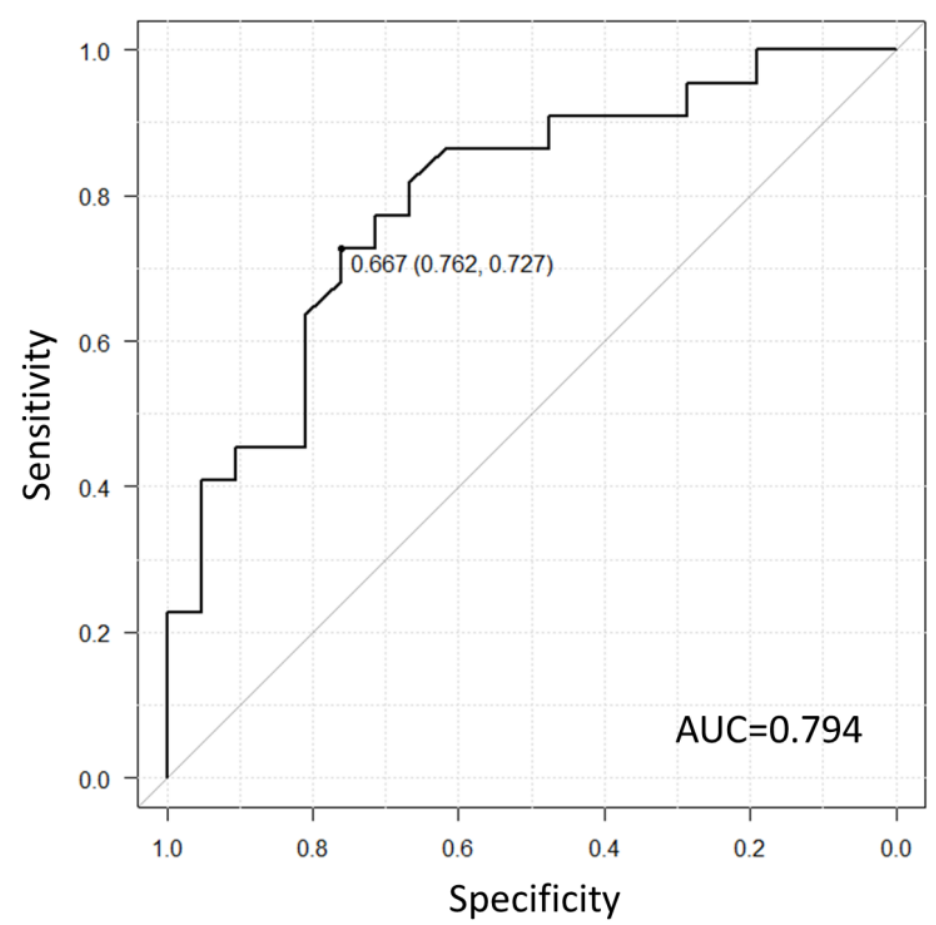

Figure 1. Receiver operating characteristic and area under the curve (AUC) of the lymphocyte-to-monocyte ratio at the time of diagnosis for all dogs. The AUC was 0.794 (95\% confidence interval, ranged between 0.659-0.930, p = 0.002).

\section{Analysis of outcomes}

The overall median TTP and OS were 46 days (ranged between 1-289 days) and 108 days (ranged between 1-621 days), respectively. At the end of data analysis, three dogs were still alive and in CR status, and survival times were 168, 226, and 248 days. Survival analysis with Kaplan-Meier and log-rank tests showed that dogs with a high LMR had significantly longer TTP $(\mathrm{p}=0.048)$ and OS $(\mathrm{p}=0.011)$ compared to dogs with a low LMR (Table 2, Figures 2 and 3 ). Moreover, immunophenotype (TTP, $\mathrm{p}=0.017$; OS, $\mathrm{p}=0.034)$, body weight (TTP, $\mathrm{p}=0.049)$, treatment $(\mathrm{TTP}, \mathrm{p}<$ 0.001; OS, $\mathrm{p}<0.001$ ), and response to treatment (TTP, p < 0.001; OS, p < 0.001) were associated with TTP and OS (Table 2, Figures 2 and 3). There were no significant differences in the TTP and OS in relation to anatomical form, clinical stage, substage, age, sex, hypercalcemia, anemia, lymphopenia, monocytosis, and prednisolone treatment prior to sampling (Table 2). 
Table 2. Analysis of prognostic factors for time to progression and overall survival in 43 dogs with high-grade lymphoma at Veterinary Medical Center of Obihiro University of Agriculture and Veterinary Medicine between 2013 and 2018.

\begin{tabular}{|c|c|c|c|c|c|}
\hline Parameters & $\begin{array}{c}\text { Case } \\
\text { (Number) }\end{array}$ & TTP* & p-value & OS* & p-value \\
\hline Age & & & 0.103 & & 0.439 \\
\hline$\geq 10$ years & 21 & $40(5-267)$ & & $71(9-621)$ & \\
\hline$<10$ years & 22 & $64(1-289)$ & & $123(1-487))$ & \\
\hline Sex & & & 0.438 & & 0.219 \\
\hline Male (Castrated) & 19 & $47.5(1-238)$ & & $97.5(1-492)$ & \\
\hline Female (Spayed) & 24 & $55(1-289)$ & & $121(19-621)$ & \\
\hline Body weight & & & 0.049 & & 0.064 \\
\hline$\geq 10 \mathrm{~kg}$ & 19 & $93(1-289)$ & & $152(7-621)$ & \\
\hline$<10 \mathrm{~kg}$ & 24 & $43.5(1-248)$ & & $68.5(1-492)$ & \\
\hline Stage & & & 0.808 & & 0.769 \\
\hline I & 3 & $28(10-267)$ & & $50(47-621)$ & \\
\hline II & 5 & $38(19-248)$ & & $61(119-248)$ & \\
\hline III & 10 & $54(8-171)$ & & $119(13-320)$ & \\
\hline IV & 16 & $45(1-289)$ & & $79(1-492)$ & \\
\hline $\mathrm{V}$ & 9 & $93(5-226)$ & & $159(9-253)$ & \\
\hline Substage & & & 0.812 & & 0.949 \\
\hline $\mathrm{a}$ & 17 & $72(1-171)$ & & $130(13-478)$ & \\
\hline $\mathrm{b}$ & 26 & $45(1-289)$ & & $68.5(1-621)$ & \\
\hline Immunophenotype $\dagger$ & & & 0.017 & & 0.034 \\
\hline B cell & 20 & 73.5 (1-289) & & $136(1-621)$ & \\
\hline $\mathrm{T}$ cell & 17 & $43(1-248)$ & & $66(9-478)$ & \\
\hline Null type & 4 & $25(1-44)$ & & $46(7-105)$ & \\
\hline Anatomical form & & & 0.123 & & 0.186 \\
\hline Multicentric & 24 & 73.5 (1-289) & & $136(1-621)$ & \\
\hline Alimentary & 11 & $43(1-135)$ & & $66(7-253)$ & \\
\hline Cutaneous & 5 & $44(1-107)$ & & $105(32-478)$ & \\
\hline Others (renal, CNS, mediastinal) & 3 & $10(5-248)$ & & $47(9-248)$ & \\
\hline Hypercalcemia & & & 0.838 & & 0.959 \\
\hline Yes & 4 & $42(19-238)$ & & $60.5(19-492)$ & \\
\hline No & 39 & $49(1-289)$ & & $121(1-621)$ & \\
\hline Presence of anemia & & & 0.287 & & 0.670 \\
\hline Yes & 16 & $50.5(1-289)$ & & $104(1-492)$ & \\
\hline No & 27 & $49(1-267)$ & & $108(13-621)$ & \\
\hline Lymphopenia & & & 0.480 & & 0.428 \\
\hline$\geq 1000$ cells $/ \mu 1$ & 25 & $63(1-289)$ & & $130(1-492)$ & \\
\hline$<1000$ cells $/ \mu 1$ & 18 & $45(1-264)$ & & $68.5(7-621)$ & \\
\hline Monocytosis & & & 0.201 & & 0.076 \\
\hline$\geq 1400$ cells $/ \mu 1$ & 26 & $43.5(1-289)$ & & $66(1-492)$ & \\
\hline$<1400$ cells $/ \mu 1$ & 17 & $83(1-267)$ & & $134(19-621)$ & \\
\hline Treatment & & & $<0.001$ & & $<0.001$ \\
\hline CHOP-based & 27 & $63(1-289)$ & & $134(1-621)$ & \\
\hline Single agent & 10 & $49(1-135)$ & & $106.5(47-478)$ & \\
\hline Prednisolonealone & 6 & $15(1-38)$ & & $22(7-61)$ & \\
\hline Response to treatment & & & $<0.001$ & & $<0.001$ \\
\hline $\mathrm{CR}$ & 16 & $147(28-289)$ & & $250(43-621)$ & \\
\hline PR & 18 & $47.5(13-132)$ & & $96(13-478)$ & \\
\hline NR & 9 & $8(1-52)$ & & $18(1-66)$ & \\
\hline Prior administration of prednisolone & & & 0.271 & & 0.126 \\
\hline Yes & 8 & $47.5(10-107)$ & & $68.5(47-138)$ & \\
\hline No & 35 & $52(0-289)$ & & $121(1-621)$ & \\
\hline LMR & & & 0.048 & & 0.011 \\
\hline$\geq 0.7$ & 22 & $79(1-289)$ & & $148(1-621)$ & \\
\hline$<0.7$ & 21 & $43(1-248)$ & & $61(9-492)$ & \\
\hline
\end{tabular}

*: Median (range); $\dagger: 2$ dogs were excluded because of inadequate data. TTP: Time To Progression; OS: Overall Survival; LMR: Lymphocyte-toMonocyte Ratio; CHOP: Cyclophosphamide, Doxorubicin, Vincristine, and Prednisone; CNS: Central Nervous System; CR: Complete Remission; PR: Partial Remission; NR: No Remission. 

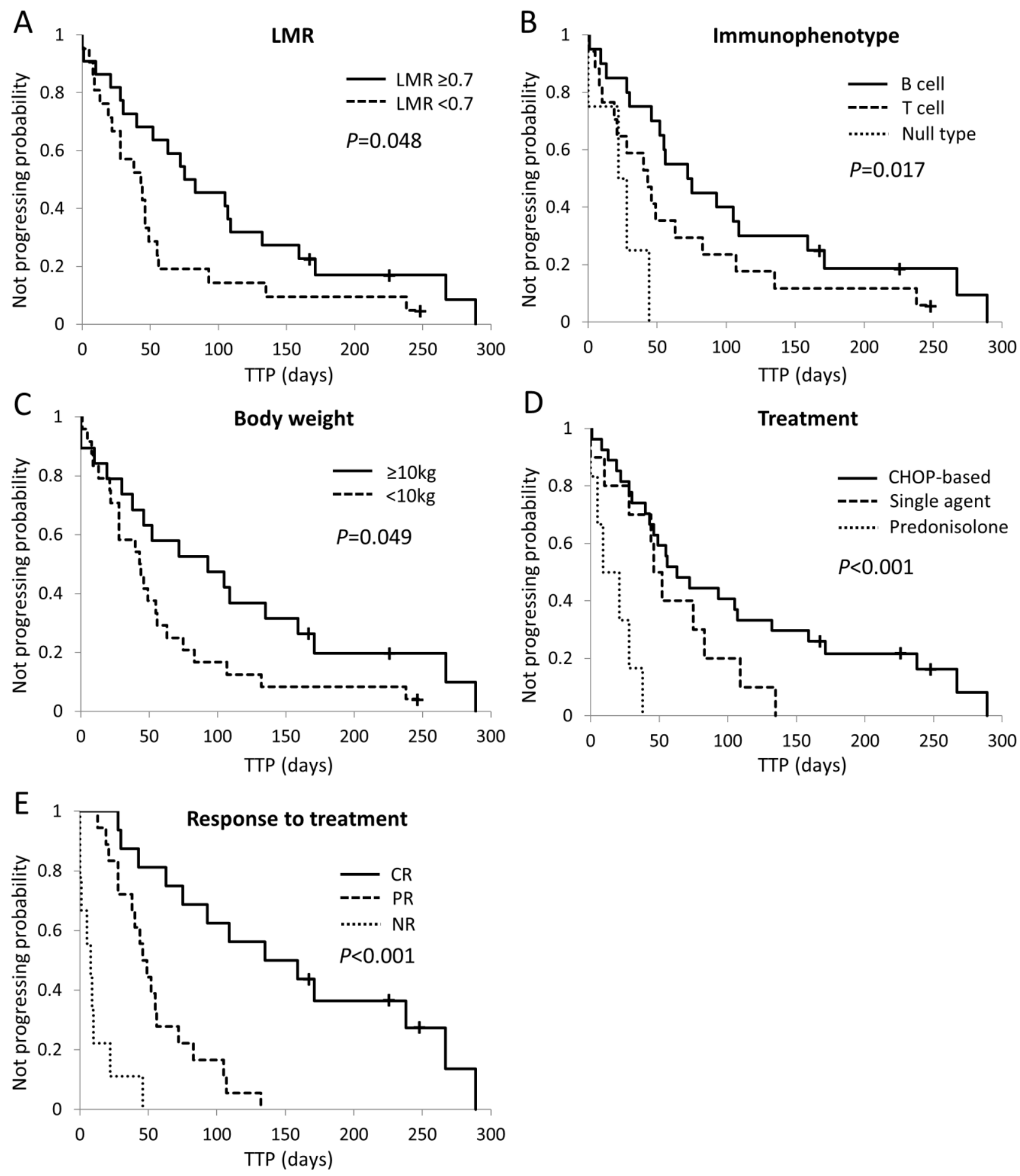

Figure 2. Kaplan-Meier curves of Time To Progression (TTP) for 43 dogs with high-grade lymphoma according to Lymphocyte-to- Monocyte Ratio (LMR) (A), immunophenotype (B), body weight (C), treatment (D), and response to treatment (E). +: censored case; CHOP: Cyclophosphamide, Doxorubicin, Vincristine, and Prednisone. 

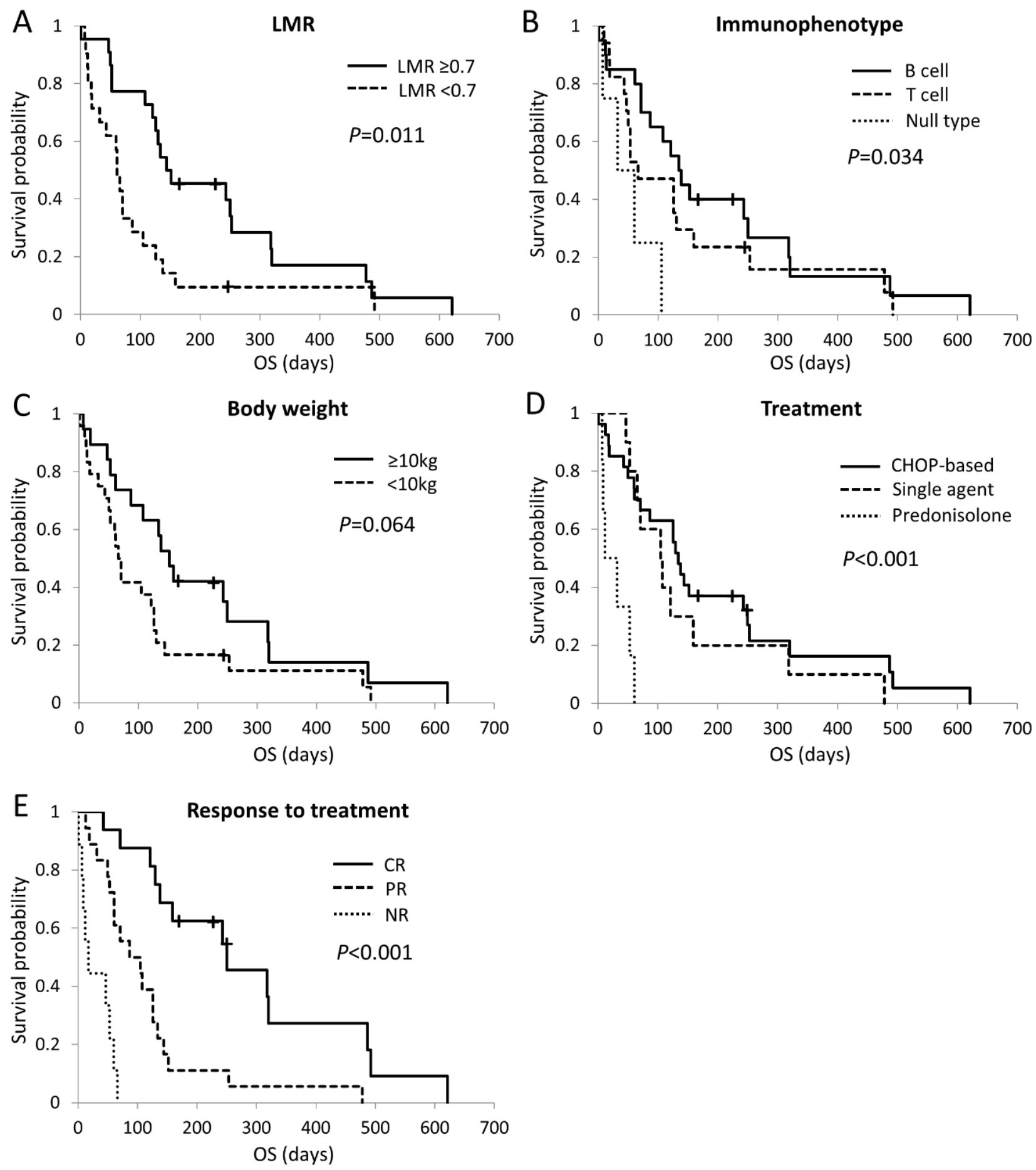

Figure 3. Kaplan-Meier curves of Overall Survival (OS) for 43 dogs with high-grade lymphoma according to Lymphocyte-to- Monocyte Ratio (LMR) (A), immunophenotype (B), body weight (C), treatment (D), and response to treatment (E). +: censored case; CHOP: Cyclophosphamide, Doxorubicin, Vincristine, and Prednisone.

To develop a combined model for the prognosis of patients with high-grade lymphoma, the associations between patient prognosis and LMR ( $\geq$ or $<0.7$ ), immunophenotype (B-cell or other types), anatomical form (multicentric or other types), body weight ( $\geq$ or $<10 \mathrm{~kg}$ ), monocytosis $(\geq$ or $<1400$ cells $/ \mu \mathrm{l})$, treatment (CHOP and single agent or prednisolone alone), and response to treatment (CR or other), which were all $\mathrm{p} \leq 0.1$ parameters in the univariate analysis, were evaluated using multivariate Cox analysis. The multivariate analysis showed that treatment $(\mathrm{p}=0.048)$ and response to treatment $(\mathrm{p}<0.001)$ were independent risk factors for the TTP $($ Table 3$)$. Moreover, the LMR $(\mathrm{p}=$ $0.020)$, treatment $(\mathrm{p}=0.029)$ and response to treatment $(\mathrm{p}<0.001)$ were independent predictors of OS (Table 4$)$. 
Table 3. Univariate and multivariate analysis of prognostic factors for time to progression of 43 dogs with high-grade lymphoma at Veterinary Medical Center of Obihiro University of Agriculture and Veterinary Medicine between 2013 and 2018.

\begin{tabular}{|c|c|c|c|c|}
\hline \multirow{2}{*}{ Parameters } & \multicolumn{2}{|c|}{ Univariate analysis } & \multicolumn{2}{|c|}{ Multivariate analysis } \\
\hline & p-value & HR $(95 \% \mathrm{CI})$ & p-value & HR $(95 \% \mathrm{CI})$ \\
\hline \multicolumn{5}{|l|}{ LMR } \\
\hline$\geq 0.7$ & 0.053 & & 0.118 & \\
\hline$<0.7$ & & $1.900(0.991-3.672)$ & & $1.887(0.851-4.259)$ \\
\hline \multicolumn{5}{|l|}{ Immunophenotype } \\
\hline B cell & 0.058 & & 0.982 & \\
\hline Other* & & $1.867(0.979-3.639)$ & & $0.990(0.411-2.439)$ \\
\hline \multicolumn{5}{|l|}{ Anatomic form } \\
\hline Multicentric & 0.051 & & 0.834 & \\
\hline Other** & & $1.930(0.997-3.714)$ & & $0.920(0.421-2.037)$ \\
\hline \multicolumn{5}{|l|}{ Body weight } \\
\hline$\geq 10 \mathrm{~kg}$ & 0.051 & & 0.984 & \\
\hline$<10 \mathrm{~kg}$ & & $1.924(0.998-3.823)$ & & $0.991(0.404-2.329)$ \\
\hline \multicolumn{5}{|l|}{ Treatment } \\
\hline $\mathrm{CHOP}$ and single agent & 0.001 & & 0.048 & \\
\hline Prednisolone alone & & $6.633(2.215-18.279)$ & & 3.277 (1.013-9.915) \\
\hline \multicolumn{5}{|l|}{ Response to treatment } \\
\hline $\mathrm{CR}$ & $<0.001$ & & $<0.001$ & \\
\hline PR, NR & & $6.352(2.780-16.545)$ & & $6.331(2.430-18.406)$ \\
\hline
\end{tabular}

Table 4. Univariate and multivariate analyses of prognostic factors for overall survival of 43 dogs with high-grade lymphoma at Veterinary Medical Center of Obihiro University of Agriculture and Veterinary Medicine between 2013 and 2018.

\begin{tabular}{|c|c|c|c|c|}
\hline \multirow{2}{*}{ Parameters } & \multicolumn{2}{|c|}{ Univariate analysis } & \multicolumn{2}{|c|}{ Multivariate analysis } \\
\hline & p-value & HR $(95 \%$ CI $)$ & p-value & HR $(95 \%$ CI) \\
\hline \multicolumn{5}{|l|}{ LMR } \\
\hline$\geq 0.7$ & 0.014 & & 0.020 & \\
\hline$<0.7$ & & $2.264(1.179-4.363)$ & & $2.551(1.161-5.753)$ \\
\hline \multicolumn{5}{|l|}{ Anatomical form } \\
\hline Multicentric & 0.056 & & 0.360 & \\
\hline Other* & & $1.909(0.983-3.694)$ & & $0.703(0.330-1.501)$ \\
\hline \multicolumn{5}{|l|}{ Body weight } \\
\hline$\geq 10 \mathrm{~kg}$ & 0.069 & & 0.837 & \\
\hline$<10 \mathrm{~kg}$ & & $1.824(0.962-3.545)$ & & $0.926(0.446-1.946)$ \\
\hline \multicolumn{5}{|l|}{ Monocytosis } \\
\hline$\geq 1400$ cells $/ \mu 1$ & 0.076 & & 0.479 & \\
\hline$<1400$ cells $/ \mu 1$ & & $0.557(0.280-1.061)$ & & $0.766(0.359-1.602)$ \\
\hline \multicolumn{5}{|l|}{ Treatment } \\
\hline $\mathrm{CHOP}$ and single agent & $<0.001$ & & 0.029 & \\
\hline Prednisolone alone & & $7.712(2.565-21.330)$ & & $3.635(1.149-10.754)$ \\
\hline \multicolumn{5}{|l|}{ Response to treatment } \\
\hline $\mathrm{CR}$ & $<0.001$ & & $<0.001$ & \\
\hline PR, NR & & $4.421(2.137-9.867)$ & & $5.858(2.259-16.172)$ \\
\hline
\end{tabular}


Prognostic factors for patients with cancer provide information regarding possible clinical outcomes and help to classify patients into different risk groups. In dogs, several consistent prognostic factors for lymphoma, including WHO clinical stage and substage, immunophenotype, anatomical form, hypercalcemia and anemia, have been reported (Rassnick et al., 2009; Parihar et al., 2010; Marconato et al., 2011). A low LMR has been reported to be a prognostic factor in human patients with hematologic malignancies (Failing et al., 2017). Although recent findings indicated that the LMR might predict the clinical outcome of canine diffuse large B-cell lymphoma (Marconato et al., 2015; Davies et al., 2018), there are limited data available on the prognostic value of the LMR for various types of high-grade lymphoma. The current study retrospectively investigated the association between clinicopathological variables, including the LMR and patient outcome of 43 dogs with high-grade lymphoma.

In the present study, log-rank tests revealed that dogs with a low LMR $(\leq 0.7)$ had significantly shorter TTP and OS than those had a high LMR (>0.7). The actual mechanisms concerning the relationship between low LMR and poor patient outcome are unclear. There are several possible explanations accounting for this correlation. Lymphocytes are the basic components of antitumor immunity and tumor-infiltrating lymphocytes have a vital effect on tumor development (Rahir and Moser, 2012). Several studies have shown that lymphopenia was associated with poor prognosis (Talaulikar et al., 2008; Castillo et al., 2010). Monocytes play a vital role in tumor progression. These cells are recruited to the tumor stroma and differentiated into Tumor-associated macrophages (TAMs) (Richards et al., 2013). TAMs have also been implicated in promoting tumor invasion and angiogenesis as well as having immunosuppressive effects on the anti-tumor response of lymphocytes by producing various cytokines and chemokines (Nielsen and Schmid, 2017). TAM infiltration has also been associated with vascular endothelial growth factor expression and linked to poor prognosis in canine breast carcinoma (Raposo et al., 2014; Raposo et al., 2015). It is suggested that the peripheral monocyte level correlates with TAM density of the tumor microenvironment (Shibutani et al., 2017). Briefly, the LMR is a simple biomarker that reflects the status of immune homeostasis and the tumor microenvironment. In the present study, an optimal cut-off value of LMR (0.7) was used for further analysis. The cut-off value of this study was lower than the LMR cut-off values reported in two previous studies on canine lymphoma (Marconato et al., 2015; Davies et al., 2018). These divergent results may have arisen for a number of reasons. The methods for calculating the percentages of lymphocytes and monocytes were different between present study and one of the aforementioned studies. In addition, the mentioned studies only analyzed diffuse large B-cell multicentric lymphoma, whereas this study included various lymphoma types. Moreover, in this study, dogs that were pretreated with prednisolone were not excluded from the leukocyte count.

In animals, lymphopenia occurs in response to endogenous or exogenous glucocorticoids. It has been reported that lymphocyte counts were significantly decreased compared to those of healthy control dogs after two weeks of antiinflammatory doses of prednisone therapy (Moore et al., 1992). However, it is reported that prednisone administration to healthy dogs with allergic dermatitis caused no significant changes in lymphocyte concentration (Masters et al., 2018). In this study, eight dogs treated with prednisolone prior to sampling had lower lymphocyte concentrations (median 555 cells $/ \mu 1$; ranged between 0-1944 cells/ $\mu 1$ ) compared to the remaining dogs (median $1395 / \mu 1$; ranged between 0-12376 cells/ $\mu \mathrm{l})$. Due to the small sample size, it is difficult to assess whether the lymphocyte concentrations of these eight dogs were affected by prednisone administration or not. In the studied cohort of dogs, lymphopenia at diagnosis was not found to be a significant prognostic factor for the TTP and OS. In addition, the prognostic significance of lymphopenia has not been documented in veterinary medicine. For further prospective studies of assessing lymphopenia as a prognostic factor, it is suggested that a history of prior glucocorticoid should be an exclusion criterion.

The log-rank tests also indicated that immunophenotype, body weight, treatment, and response to treatment were associated with patient outcome. Dogs with B-cell lymphoma were found to have longer TTP and OS compared to those with T-cell lymphoma or null type. In general, T-cell lymphomas have shorter remission and survival times than B-cell lymphomas (Zandvliet, 2016). It has been reported that some subsets of T-cells have 40 times more P-glycoprotein gene expression than B-cells (Klimecki et al., 1994). The lack of response to chemotherapy in T-cell lymphomas may be partially explained by their multidrug resistance. In fact, intrinsic drug resistance was more common in T-cell than B-cell lymphoma (Zandvliet, 2016). Moreover, extranodal lymphomas, including gastrointestinal, hepatosplenic, mediastinal, cutaneous, and renal lymphoma, have poorer prognoses than the multicentric form (Zandvliet, 2016). However, in the present study, no significant differences in the TTP and OS in relation to anatomical form were found. This contradiction can be attributed to small sample size. In addition, $28 \%$ of multicentric lymphomas had T-cell immunophenotype which is a negative prognostic factor. The log-rank test showed that dogs with body weights $\geq 10 \mathrm{~kg}$ were found to have significantly longer TTP and a longer OS tendency. Marconato et al. (2011) studied the predictors of long-term survival in Rottweilers, Pointer, Boxer, Yorkshire Terrier, Bernese Mountain Dog, and Doberman Pinscher dogs with high-grade multicentric lymphoma. The mentioned study found that $11 / 13 \mathrm{dogs}$ that were long-term survivors had body weights $\geq$ $10 \mathrm{~kg}$. In addition, excessive body weight in dogs has been associated with positive outcomes (Romano et al., 2016). More research is needed to elucidate the relationship between body weight and prognosis in dogs with lymphoma. In 
veterinary medicine, various chemotherapies have been described, and multi-agent therapy protocols including CHOP have the highest response rate and longest response durations for treating high-grade lymphoma (Castillo et al., 2010; Valli et al., 2013). It is thought that single-agent therapies are less effective than a doxorubicin-based multi-agent protocol and should be reserved for palliative therapy (Sauerbrey et al., 2007; Higginbotham et al., 2013). However, several single-agent protocols have shown no significant differences in remission or survival time compared to multidrug treatments (Simon et al., 2008; Deravi et al., 2017). In this study, the TTP and OS were substantially longer in dogs treated with chemotherapy protocols versus prednisolone alone. Response to treatment has been a strong prognostic factor in several case studies of canine lymphoma (Curran and Thamm, 2016; Goodman et al., 2016). The present study also revealed that dogs reaching $\mathrm{CR}$ as best response to therapy had significantly longer survival times compared to the other dogs. According to multivariate analysis of data from dogs with high-grade lymphoma, treatment regimen and response to treatment remained significant for the TTP. Moreover, the LMR, treatment, and response to treatment were independent prognostic factors. In particular, the LMR may be useful in clinical practice as a simple and readily available prognostic marker since it can only predict the outcome in pre-treatment dogs with high-grade lymphoma.

\section{CONCLUSION}

A low LMR was significantly associated with a poor prognosis in dogs with different types of high-grade lymphoma. The current study had several limitations. First, the small sample size for each analysis may limit the detection of differences between groups. Second, the present study was a retrospective analysis and first-line and rescue treatments were not standardized. The choice of treatment mainly depended on the owner's opinion and the patient's condition. Thus, future prospective studies which using standardized treatment are necessary for adequate evaluation. Further prospective studies and analysis of the LMR levels obtained from routine blood tests may provide additional information to assist in the management of dogs with high-grade lymphoma.

\section{DECLARATIONS}

\section{Acknowledgments}

The authors thank all veterinary medical staff of the Veterinary Medical Center, Obihiro University of Agriculture and Veterinary Medicine for kindly and diligently collecting the patient information.

\section{Authors' contributions}

MT designed the study and drafted the manuscript, GS and KM performed the practical part of the experiment. KM reviewed the manuscript. All the authors approved the final manuscript.

\section{Consent to publish}

All the authors approved and agreed to publish the manuscript.

\section{Competing interests}

The authors clarify that they have no competing interest, and with respect to this search, all the authors are in agreement with each other and have no conflict with authorship or article publication, all authors approved the publishing of the paper.

\section{REFERENCES}

Aoki K, Tabata S, Yonetani N, Matsushita A and Ishikawa T (2013). The prognostic impact of absolute lymphocyte and monocyte counts at diagnosis of diffuse large B-cell lymphoma in the rituximab era. Acta Haematologica, 130: 242-246. DOI: https://doi.org/10.1159/000350484

Argyle DJ and Pecceu E (2016). Canine and feline lymphoma: challenges and opportunities for creating a paradigm shift. Veterinary and Comparative Oncology, 14: 1-7. DOI: https://doi.org/10.1111/vco.12253

Burton JH, Garrett-Mayer E and Thamm DH (2013). Evaluation of a 15-week CHOP protocol for the treatment of canine multicentric lymphoma. Veterinary and Comparative Oncology, 11: 306-315. DOI: https://doi.org/10.1111/j.1476-5829.2012.00324.x

Castillo JJ, Morales D, Quinones P, Cotrina E, Desposorio C and Beltran B (2010). Lymphopenia as a prognostic factor in patients with peripheral T-cell lymphoma, unspecified. Leukemia and Lymphoma, 51: 1822-1828. DOI: https://doi.org/10.3109/10428194.2010.508189

Childress MO, Ramos-Vara JA and Ruple A (2018). Retrospective analysis of factors affecting clinical outcome following CHOPbased chemotherapy in dogs with primary nodal diffuse large B-cell lymphoma. Veterinary and Comparative Oncology, 16: E159-E168. DOI: https://doi.org/10.1111/vco.12364

Curran K and Thamm DH (2016). Retrospective analysis for treatment of naïve canine multicentric lymphoma with a 15-week, maintenance-free CHOP protocol. Veterinary and Comparative Oncology, 14 (supplement 1): 147-155. DOI: https://doi.org/10.1111/vco.12163 
Davies O, Szladovits B, Polton G, Garden OA, Leo C and Lara-Garcia A (2018). Prognostic significance of clinical presentation, induction and rescue treatment in 42 cases of canine centroblastic diffuse large B-cell multicentric lymphoma in the United Kingdom. Veterinary and Comparative Oncology, 16: 276-287. DOI: https://doi.org/10.1111/vco.12378

Deravi N, Berke O, Woods JP and Bienzle D (2017). Specific immunotypes of canine T cell lymphoma are associated with different outcomes. Veterinary Immunology and Immunopathology, 191: 5-13. DOI: https://doi.org/10.1016/j.vetimm.2017.07.008

Failing JJ, Yan Y, Porrata LF and Markovic SN (2017). Lymphocyte-to-monocyte ratio is associated with survival in pembrolizumabtreated metastatic melanoma patients. Melanoma Research, 27: 596-600. DOI: https://doi.org/10.1097/CMR.0000000000000404

Fournel-Fleury C, Magnol JP, Bricaire P, Marchal T, Chabanne L, Delverdier A, Bryon PA and Felman P (1997). Cytohistological and immunological classification of canine malignant lymphomas: comparison with human non-Hodgkin's lymphomas. Journal of Comparative Pathology, 117: 35-59. DOI: https://doi.org/10.1016/S0021-9975(97)80065-5

Goodman IH, Moore AS and Frimberger AE (2016). Treatment of canine non-indolent T cell lymphoma using the VELCAP-TSC protocol: A retrospective evaluation of $70 \mathrm{dogs}$ (2003-2013). The Veterinary Journal, 211: 39-44. DOI: https://doi.org/10.1016/j.tvj1.2016.03.003

Graff EC, Spangler EA, Smith A, Denhere M and Brauss M (2014). Hematologic findings predictive of bone marrow disease in dogs with multicentric large-cell lymphoma. Veterinary Clinical Pathology, 43: 505-512. DOI: https://doi.org/10.1111/vcp.12182

Higginbotham ML, McCaw DL, Roush JK, Nietfeld JC, Wilkerson MJ, Reeds K and Burr D (2013). Intermittent single-agent doxorubicin for the treatment of canine B-cell lymphoma. Journal of the American Animal Hospital Association, 49: $357-362$. DOI: https://doi.org/10.5326/JAAHA-MS-5929

Klimecki WT, Futscher BW, Grogan TM and Dalton WS (1994). P-glycoprotein expression and function in circulating blood cells from normal volunteers. Blood, 83: 2451-2458.

Lana SE, Jackson TL, Burnett RC, Morley PS and Avery AC (2006). Utility of polymerase chain reaction for analysis of antigen receptor rearrangement in staging and predicting prognosis in dogs with lymphoma. Journal of Veterinary Internal Medicine, 20: 329-334. DOI: https://doi.org/10.1111/j.1939-1676.2006.tb02864.x

Marconato L, Martini V, Stefanello D, Moretti P, Ferrari R, Comazzi S, Laganga P, Riondato F and Aresu L (2015). Peripheral blood lymphocyte/monocyte ratio as a useful prognostic factor in dogs with diffuse large B-cell lymphoma receiving chemoimmunotherapy. The Veterinary Journal, 206: 226-230. DOI: https://doi.org/10.1016/j.tvj1.2015.07.009

Marconato L, Stefanello D, Valenti P, Bonfanti U, Comazzi S, Roccabianca P, Caniatti M, Romanelli G, Massari F and Zini E (2011). Predictors of long-term survival in dogs with high-grade multicentric lymphoma. Journal of the American Veterinary Medical Association, 238: 480-485. DOI: https://doi.org/10.2460/javma.238.4.480

Masters AK, Berger DJ, Ware WA, Langenfeld NR, Coetzee JF, Mochel JPM and Ward JL (2018). Effects of short-term antiinflammatory glucocorticoid treatment on clinicopathologic, echocardiographic, and hemodynamic variables in systemically healthy dogs. American Journal of Veterinary Research, 79: 411-423. DOI: https://doi.org/10.2460/ajvr.79.4.411

Moore GE, Mahaffey EA and Hoenig M (1992). Hematologic and serum biochemical effects of long-term administration of antiinflammatory doses of prednisone in dogs. American Journal of Veterinary Research, 53: 1033-1037.

Mutz M, Boudreaux B, Kearney M, Stroda K, Gaunt S and Shiomitsu K (2015). Prognostic value of baseline absolute lymphocyte concentration and neutrophil/lymphocyte ratio in dogs with newly diagnosed multi-centric lymphoma. Veterinary and Comparative Oncology, 13: 337-347. DOI: https://doi.org/10.1111/vco.12045

Nielsen SR and Schmid MC (2017). Macrophages as key drivers of cancer progression and metastasis. Mediators of Inflammation, 2017: 9624760. DOI: https://doi.org/10.1155/2017/9624760

Owen LN (1980). TNM Classification of Tumours in Domestic Animals. Geneva, Switzerland: World Health Organization. Available at: https://apps.who.int/iris/handle/10665/68618

Parihar A, Eubank TD and Doseff AI (2010). Monocytes and macrophages regulate immunity through dynamic networks of survival and cell death. Journal of Innate Immunity, 2: 204-215. DOI: https://doi.org/10.1159/000296507

Rahir G and Moser M (2012). Tumor microenvironment and lymphocyte infiltration. Cancer Immunology and Immunotherapy, 61: 751-759. DOI: https://doi.org/10.1007/s00262-012-1253-1

Raposo T, Gregório H, Pires I, Prada J and Queiroga FL (2014). Prognostic value of tumour-associated macrophages in canine mammary tumours. Veterinary and Comparative Oncology, 12: 10-19. DOI: https://doi.org/10.1111/j.1476-5829.2012.00326.x

Raposo TP, Pires I, Carvalho MI, Prada J, Argyle DJ and Queiroga FL (2015). Tumour-associated macrophages are associated with vascular endothelial growth factor expression in canine mammary tumours. Veterinary and Comparative Oncology, 13: 464-474. DOI: https://doi.org/10.1111/vco.12067

Rassnick KM, Moore AS, Collister KE, Northrup NC, Kristal O, Chretin JD and Bailey DB (2009). Efficacy of combination chemotherapy for treatment of gastrointestinal lymphoma in dogs. Journal of Veterinary Internal Medicine, 23: 317-322. DOI: https://doi.org/10.1111/j.1939-1676.2008.0270.x

Ray-Coquard I, Cropet C, Van Glabbeke M, Sebban C, Le Cesne A, Judson I, Tredan O, Verweij J, Biron P, Labidi I, et al. (2009). Lymphopenia as a prognostic factor for overall survival in advanced carcinomas, sarcomas, and lymphomas. Cancer Research, 69: 5383-5391. DOI: https://doi.org/10.1158/0008-5472.CAN-08-3845

Richards DM, Hettinger J and Feuerer M (2013). Monocytes and macrophages in cancer: development and functions. Cancer Microenvironment, 6: 179-191. DOI: https://doi.org/10.1007/s12307-012-0123-x

Romano FR, Heinze CR, Barber LG, Mason JB and Freeman LM (2016). Association between body condition score and cancer prognosis in dogs with lymphoma and osteosarcoma. Journal of Veterinary Internal Medicine, 30: 1179-1186. DOI: https://doi.org/10.1111/jvim.13965 
Sauerbrey ML, Mullins MN, Bannink EO, Van Dorp TE, Kaneene JB and Obradovich JE (2007). Lomustine and prednisone as a firstline treatment for dogs with multicentric lymphoma: 17 cases (2004-2005). Journal of American Veterinary Medical Association, 230: 1866-1869. DOI: https://doi.org/10.2460/javma.230.12.1866

Shibutani M, Maeda K, Nagahara H, Fukuoka T, Nakao S, Matsutani S, Hirakawa K and Ohira M (2017). The peripheral monocyte count is associated with the density of tumor-associated macrophages in the tumor microenvironment of colorectal cancer: a retrospective study. BioMed Central Cancer, 17: 404. DOI: https//doi.org/10.1186/s12885-017-3395-1

Simon D, Moreno SN, Hirschberger J, Moritz A, Kohn B, Neumann S, Jurina K, Scharvogel S, Schwedes C, Reinacher M, et al. (2008). Efficacy of a continuous, multiagent chemotherapeutic protocol versus a short-term single-agent protocol in dogs with lymphoma. Journal of American Veterinary Medical Association, 232: 879-885. DOI: https//doi.org/10.2460/javma.232.6.879

Tagawa M, Kurashima C, Takagi S, Maekawa N, Konnai S, Shimbo G, Matsumoto K, Inokuma H, Kawamoto K and Miyahara K (2018). Evaluation of costimulatory molecules in dogs with B cell high grade lymphoma. PLoS One, 13: e0201222. DOI: https://doi.org/10.1371/journal.pone.0201222

Talaulikar D, Choudhury A, Shadbolt B and Brown M (2008). Lymphocytopenia as a prognostic marker for diffuse large B cell lymphomas. Leukemia and Lymphoma, 49: 959-964. DOI: https://doi.org/10.1080/10428190801959026

Teske E, van Heerde P, Rutteman GR, Kurzman ID, Moore PF and MacEwen EG (1994). Prognostic factors for treatment of malignant lymphoma in dogs. Journal of American Veterinary Medical Association, 2015: 1722-1728.

Vail DM, Michels GM, Khanna C, Selting KA and London CA; Veterinary Cooperative Oncology Group (2010). Response evaluation criteria for peripheral nodal lymphoma in dogs (v1.0)-a Veterinary Cooperative Oncology Group (VCOG) consensus document. Veterinary and Comparative Oncology, 8: 28-37. DOI: https://doi.org/10.1111/j.1476-5829.2009.00200.x

Vail DM, Pinkerton ME, Young KM (2013). Hematopoietic tumors. In: SJ Withrow and DM Vail (Editors), Withrow and MacEwen's Small Animal Clinical Oncology, 5th Edition. Saunders Elsevier, St. Louis, MO. pp. 608-678.

Valli VE, Kass PH, San Myint M and Scott F (2013). Canine lymphomas: association of classification type, disease stage, tumor subtype, mitotic rate, and treatment with survival. Veterinary Pathology, 50: 738-748. DOI: https://doi.org/10.1177/0300985813478210

Whiteside TL (2005). Lymphocyte homeostasis and the antitumor immune response. Expert Review of Clinical Immunology, 1: 369378. DOI: https://doi.org/10.1586/1744666X.1.3.369

Zandvliet M (2016). Canine lymphoma: a review. The Veterinary Quarterly, 36: 76-104. DOI: https://doi.org/10.1080/01652176.2016.1152633 